\title{
Relationship between work-related ocular events, facial injuries and associated factors amongst dental professionals during COVID-19
}

\author{
Wajiha Anzar ${ }^{\mathrm{a}}$, Ashar Afaq ${ }^{\mathrm{a}}$, Qaiser Ali Baig ${ }^{\mathrm{a}}$, Osama Y. Mohamed ${ }^{\mathrm{b}}$, \\ Ishfaq A Bukhari ${ }^{\mathrm{b}}$ and Fahim Vohra ${ }^{\mathrm{c}, *}$ \\ ${ }^{a}$ Dow International Dental College, Dow University of Health Sciences Karachi, Pakistan \\ ${ }^{\mathrm{b}}$ Department of Pharmacology, College of Medicine, King Saud University, Riyadh, Saudi Arabia \\ ${ }^{\mathrm{c}}$ Department of Prosthetic Dental Science, College of Dentistry, King Saud University, Riyadh, Saudi Arabia
}

Received 13 November 2020

Accepted 12 April 2021

\begin{abstract}
.
BACKGROUND: In dental settings, COVID-19 can be transmitted directly from patients to dentists through small droplets, saliva splashes, blood, and other body fluids liberated as a result of dental procedures.

OBJECTIVE: To determine the prevalence of ocular and facial injuries in dental professionals and to investigate factors in dental practice contributing to ocular injuries.

METHODS: An analytical cross-sectional study was performed in public and private sector universities. The study had 301 participants including final year undergraduate students, interns, postgraduate trainees, general practitioners, and dental specialists. Data were gathered online using Google forms. Information on sociodemographic, practice details, history of ocular and facial encounters during the clinical experience, and protective measures adopted by the dentists were collected. Means and standard deviations were calculated for continuous variables whereas frequencies and percentages were calculated for categorical variables. A Chi-square test was applied for association between variables.

RESULTS: Ocular events and facial injuries occurred more in females $204(67.8 \%)$ than in males 97(32.2\%). Final year students reported more incidence of ocular encounters than specialists $(40.9 \%, 3.3 \%)$. Dentists working in the government sector underwent more ocular encounters than those in private sectors 185(61.4\%) and 96 (31.8\%). Majority of participants reported that scaling was the procedure in which dentists experienced an ocular event. A significant association was found between ocular events, qualification, years of experience in clinical practice, number of patients treated per day, improper posture, and proper armamentarium $(p<0.05)$. However, no association was found between ocular events, gender, working sector, and dental procedures.

CONCLUSION: Occurrence of ocular injuries were high compared to facial injuries and these outcomes were dependent on dental expertise and experiences. Appropriate measures should be adopted to minimize the risk of disease transmission and COVID-19 through the eyes among practicing dentists.
\end{abstract}

Keywords: Dental settings, aerosols, saliva, dentists, ocular, injury

*Address for correspondence: Fahim Vohra PhD, MProsRCS, MClinDent, MFDS. Professor, Department of Prosthetic Dental
Science, College of Dentistry, King Saud University, Riyadh 11545, Saudi Arabia. E-mail: fvohra@ksu.edu.sa. 


\section{Introduction}

Dental professionals exhibit many occupational diseases like systemic infections, impaired hearing, allergies, musculoskeletal disorders, injuries, and psychological problems [1,2]. In comparison to other dental professionals, dentists experience high workrelated adverse ocular events which are mainly due to the dental procedures involved in dentistry. Workrelated ocular trauma to dentists may arise from various mechanical, chemical, and microbiological sources and these injuries may result in the dentist's morbidity and disability [3].

Recent studies have reported that prevalence of ocular incidence in dentists are 63\% whereas in other dental professionals including dental technicians, dental assistants and dental nurses, it is $42.3 \%$ $[4,5]$. In dentistry, such injuries are based on the type of dental procedures and may range from instant burns, abrasion of the cornea, foreign bodies like droplets, saliva, splashes, contaminated instruments, and injuries due to penetration. When eyes get contaminated with bodily fluids like saliva, which is a reservoir for many viruses and bacteria, it poses a serious threat towards infections [2,5]. Thus, eye protection among dentists is of paramount importance. Apart from protective measures such as eyeglasses or face-shield while performing the dental treatment, use of rubber dam, proper suctioning and careful instrument handling reduces the encounters with splatter or debris [6, 7]. However, it is reported in literature that around the world a great non-compliance in dental professionals has been noted in the use of personal protective equipment (PPE) [8].

In a study by Basnet et al., 185 dental students' awareness and knowledge about eye related injuries were assessed. 95\% had sufficient knowledge related to ocular injuries that occurred during routine dental procedures like scaling, extractions, cavity preparations and as a result of foreign body encounters [9]. Oleksiak et al. reported that $88 \%$ of dentists routinely used protective glasses while performing dental treatments and $54.6 \%$ of operators provided their patients with safety glasses during treatment [1]. Furthermore, it was reported that $87 \%$ of general dental practitioners routinely used eyewear but most of the time their mode of protection was not sufficient for all forms of treatments. Surprisingly it was also seen that $75 \%$ of these injuries occur as a result of wearing eye protection [6]. Therefore, it was suggested that proper use of eyewear is critical to reduce the incidence of ocular events.
There have been improvements in the types of eyewear with the introduction of medicated goggles. In a study by Azodo et al., medicated glasses usage had a significant impact on ocular health, however, among study participants, $23 \%$ reported non-use of eye goggles and face mask [10]. A similar study concluded that ocular encounters mostly result during restorative procedures $(95 \%)$ followed by scaling and polishing (90\%), therefore suggesting that not just the use of eye wear, but also the suitability of procedure and awareness of its potential to contaminate and produce aerosol is vital for preventing ocular injuries [11].

Based on the findings of recent epidemiologic studies, it is evident that spread of 2019-nCoV, which started as animal to human transmission was later followed by human to human spread. A study by Najatindanesh et al. revealed that there was a significant difference between contamination values of different areas of face among health care professionals. Inner corners of eyes were found to be the most contaminated sites [12]. Role of oral fluids and 2019-nCoV still, has not been understood completely. In an asymptomatic patient the virus accumulates in oral, pharyngeal and nasal mucosa and binds to angiotensin converting enzyme 2 receptors [13]. Evidence have also demonstrated that droplets and bodily fluids of infected person can easily contaminate conjunctival epithelium in dentists. It is believed that presence of permissive receptors not only facilities tropism of 2019-nCoV but also allows this virus to make use of eye as a primary site of its replication and a portal of entry to extra-ocular tissues to establish clinical infestation [14].

Recently, a study performed on a mannequin with a phantom jaw which was seated on a dental chair revealed that splashes and aerosols can cover distance up to $60 \mathrm{~cm}$ from the patient's oral cavity to the eyes, nose, and mask of dentists [15]. Moreover, the aerosols which are generally generated from ultrasonic device remain suspended in the air for at least 30 minutes even after the procedure is over [16]. Therefore, dental procedures can also be considered as one of the prime factors in coronavirus transmission as these procedures require proximity with the patient's mouth and a great risk of contact with patient's saliva, mouth, and biological fluids [17-20].

In the light of the controversial evidence in the literature and the presence of Corona Virus disease 2019 (COVID-19), which is stated to be transmitted through ocular inoculation $[21,22]$, it is critical to assess the prevalence of work-related ocular events and facial injuries in dentists in relation to factors 
including type of procedures, years of practice, working sector, posture, armamentarium and workload. These findings will form the factual basis to create awareness among the dental community and to formulate corrective measures to reduce and eliminate ocular injuries and disease transmission in dentists. Therefore, the study aimed to determine the prevalence of ocular and facial injuries in dental professionals and to investigate what factors in dental practice contribute to ocular injuries.

\section{Materials and methods}

An analytical cross-sectional study was carried out in various public and private sector universities of Karachi which included Dow International Dental College, Dow Dental College, Dr. Ishratulibad Khan Institute Of oral Health Sciences, Karachi Medical and Dental College, Fatima Jinnah Dental College, Liaqat College of Medicine and dentistry and Baqai Dental College. The duration of the study was two months from August 2020 to September 2020. Ethical approval was obtained prior to start of study. The study was in accordance to STROBE statement of reporting cross sectional surveys. The sample size was calculated as 300 , considering the number of registered dentists in Karachi as 1500 at $95 \%$ confidence interval, $5 \%$ margin of error, and $80 \%$ power of study using a raosoft calculator [23]. Participants were recruited using convenience sampling technique. 400 participants were invited via email out of which 306 responded. Five drop-outs in the study were noted due to invalid response. Hence, 301 final subjects were taken as appropriate.

The study population comprised of practicing dentists of both genders regardless of race, socioeconomic status, and ethnic group. The inclusion criteria of the study were final year undergraduate students, interns, postgraduates, general dental practitioners, and specialists of respective universities who were willing to take part in the study.

Data was gathered online using Google forms. The questionnaire was adopted from a previous study with certain modifications [10]. Research team of statistician along with authors reviewed the content of each question to make sure that the survey reflected appropriate phrasing and understanding. A pilot study of the questionnaire was performed on $10 \%$ of sample size $(n=30)$ to assess validity of questionnaire. Reliability was calculated using Cronbach's alpha $(\alpha=0.70)$. The questionnaire comprised of 14 questions based on: i) sociodemographic details of study participants like age, gender and qualification, ii) practice details like working sector, years of experience, number of patients treated per day, iii) history of ocular and facial encounters during clinical experience, type of foreign body encounter, dental procedures giving rise to them, reasons for foreign body encounter, iv) protective measures adopted by dentists, v) barriers towards not using protective measures. These questions had multiple options and the participants were asked to tick the responses, this helped us to determine the prevalence of ocular events during clinical experience and to determine factors in dental practice that contribute to ocular injuries. Reminder emails were sent periodically to improve response rate.

Data was analyzed using Statistical Program for Social Sciences (SPSS) [24]. Age was taken as a continuous variable whereas other variables were recorded as categorical. Descriptive statistics (means) were calculated for continuous variables like age, whereas frequencies and percentages were obtained for categorical variables. Chi-square test was applied to look into the association between ocular encounter and factors in dental practice that contribute to ocular injuries, $p$-value $<0.05$ was considered statistically significant.

\section{Results}

A total of 301 participants took part in this study. The response rate was $76.5 \%$. Age range of participants was $19-54$, with a mean age of $24.74 \pm 2.31$. The overall prevalence of ocular encounter and the facial injury was found to be $47.8 \%$ and $5.3 \%$ respectively.

\subsection{Prevalence of ocular encounters according to sociodemographic}

Ocular encounters were more prevalent in females $204(67.8 \%)$. Another major finding was that final year students exhibited greatest frequency of ocular events $123(40.9 \%)$ followed by house officers $97(32.2 \%)$. Whereas incidence of ocular injuries was lowest among specialist 10 (3.3\%). Number of patients treated each day also had a significant role in ocular events. Outcome of current study revealed that those who treated $\leq 4$ patients per day reported the greatest frequency of work-related ocular evens [142(47.5)]. 


\subsection{Prevalence of ocular encounter according to practice areas}

Dentists working in the government sector undergo more ocular encounters than those in private sectors $(61.4 \%, 31.8 \%)$. The most common foreign body encountered by dentists was saliva (26.2\%). Forty-three percent of dentists believed that better armamentarium can reduce ocular and facial injuries. Whereas, $35 \%$ believed that improper posture is the prime contributor in such occurrences.

\subsection{Prevalence of ocular encounter according to types of injuries}

The frequencies of ocular encounters reported during various dental procedures were as follows 41 (13.6\%) during scaling, 11 (3.7\%) for polishing, 8 $(2.7 \%)$ during tooth preparation, $26(8.6 \%)$ in trimming of denture, $14(4.7 \%)$ with extraction, $10(3.3 \%)$ in amalgam removal, $28(9.3 \%)$ for irrigation, 2 $(0.7 \%)$ with suturing and $4(1.3 \%)$ were due to other procedures.

A statistically significant association was found between ocular encounters, qualification of dentists, years of experience, number of patients treated per day, improper posture and proper armamentarium $(p$-value $=0.0002,0.009,0.00001,0.043,0.003)$. However, there was no association found between ocular events, gender, working sectors, and procedures performed $(p$-value $=0.148,0.155,0.642)$. Table 1 displays the ocular encounter and their associated factors.

Approximately half of participants reported the use of protective measures like facemask in routine [144(47.8\%)] followed by combination of mask and protective eye glasses [55(18.3\%)]. Use of face shield as protective measure was among 19 (6.3\%) participants. Whereas, a very few used complete PPE which includes face mask, face shield, goggles, gloves and protective clothing. [14 (4.7\%)]. Barriers reported by dentists towards use of protective measures included unavailability, as the prime factor. This was followed by PPE which was expensive to purchase. Some dentists expressed that the use of PPE was tasking. (Table 2) (Fig. 1).

\section{Discussion}

Dental professionals have always been at great risk of cross-infection due to pathogenic microorganisms
Table 1

Ocular events and their associated factors

\begin{tabular}{|c|c|c|}
\hline & $\begin{array}{c}\text { Frequency } \\
(\%)\end{array}$ & $\begin{array}{c}\text { Chi square } \\
(p \text {-value })\end{array}$ \\
\hline \multicolumn{3}{|l|}{ Gender } \\
\hline Male & $97(32.2)$ & 0.148 \\
\hline Female & $204(67.8)$ & \\
\hline \multicolumn{3}{|l|}{ Qualification } \\
\hline Final year & $123(40.9)$ & \\
\hline House officer & $97(32.2)$ & \\
\hline Postgraduate trainee & $50(16.6)$ & 0.0002 \\
\hline General Practitioner & $21(7)$ & \\
\hline Specialist & $10(3.3)$ & \\
\hline \multicolumn{3}{|l|}{ Years of experience } \\
\hline$\leq 1$ year & $163(54.2)$ & \\
\hline $2-4$ years & $99(32.9)$ & \\
\hline $5-7$ years & $25(8.3)$ & 0.009 \\
\hline $8-10$ years & $7(2.3)$ & \\
\hline$>10$ years & $7(2.3)$ & \\
\hline \multicolumn{3}{|l|}{ Workplace } \\
\hline Private & $96(31.89)$ & \\
\hline Government & $185(61.46)$ & 0.155 \\
\hline Individual & $15(4.98)$ & \\
\hline Group & $5(1.70)$ & \\
\hline \multicolumn{3}{|l|}{ Average patients per day } \\
\hline$\leq 4$ & $142(47.2)$ & \\
\hline$\leq 8$ & $77(25.6)$ & \\
\hline$\leq 10$ & $32(10.6)$ & 0.00001 \\
\hline$>10$ & $50(16.6)$ & \\
\hline \multicolumn{3}{|l|}{ Procedure } \\
\hline Scaling & $103(34.2)$ & \\
\hline Polishing & $22(7.3)$ & \\
\hline Tooth preparation & $17(5.6)$ & \\
\hline Trimming & $53(17.6)$ & 0.642 \\
\hline Extraction & $30(10)$ & \\
\hline Amalgam removal & $16(5.3)$ & \\
\hline Irrigation & $46(15.3)$ & \\
\hline Suture & $3(1)$ & \\
\hline Others & $11(3.7)$ & \\
\hline \multicolumn{3}{|l|}{ Improper posture } \\
\hline Always & $106(35.2)$ & \\
\hline Sometimes & $128(42.5)$ & \\
\hline Occasionally & $52(17.3)$ & 0.0432 \\
\hline Rarely & $9(3)$ & \\
\hline Never & $6(2)$ & \\
\hline \multicolumn{3}{|l|}{ Proper armenterium } \\
\hline Always & $130(43.2)$ & 0.003 \\
\hline Sometimes & $119(39.5)$ & \\
\hline Occasionally & $19(6.3)$ & \\
\hline Rarely & $22(7.3)$ & \\
\hline Never & $11(3.7)$ & \\
\hline
\end{tabular}

and viruses derived from the oral cavity and airways [15]. This group of professionals on daily basis experience contagion and infection transmission as the oral environment contains high levels of microbes which are liberated as aerosols due to dental procedures $[25,26]$. Recently, the possible transmission of 2019-nCoV has been suggested by three major pathways. Firstly, due to direct exposure to contaminated, droplets, saliva splashes during 


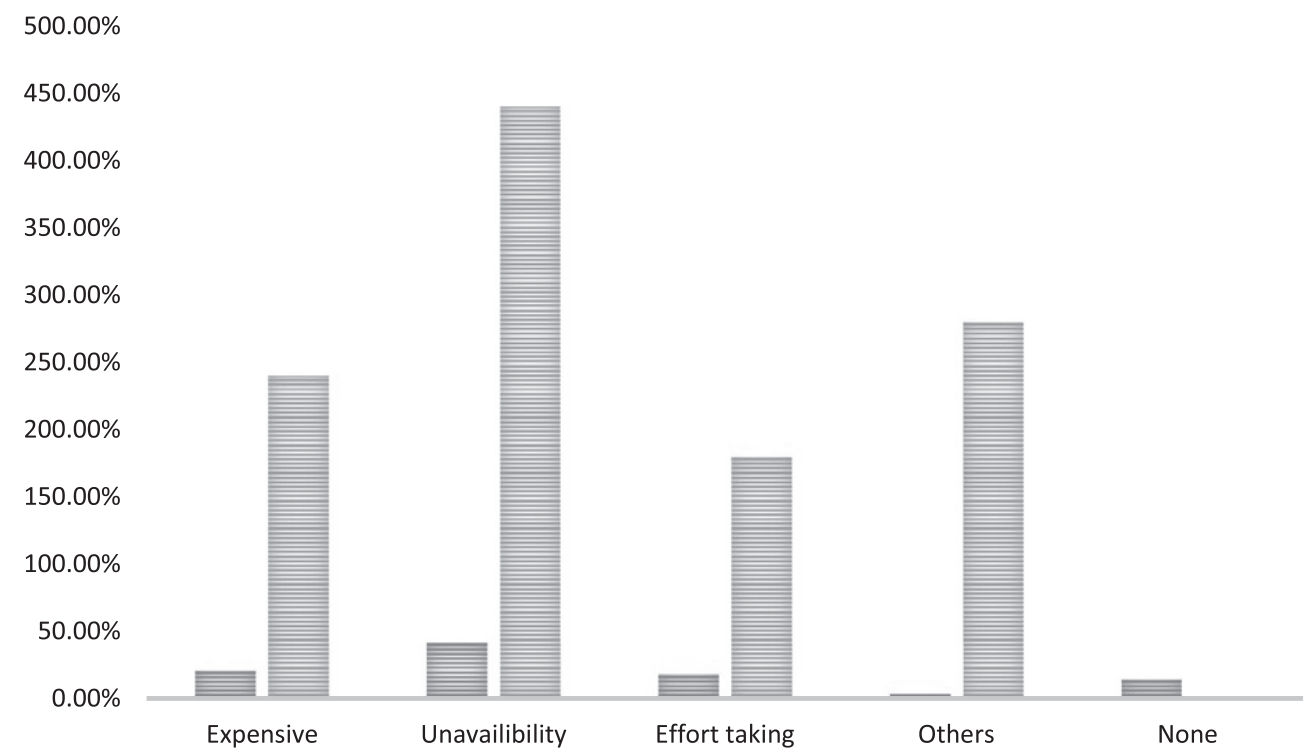

Fig. 1. Barriers towards use of protective measures.

Table 2

Protective measures adopted by dentists

\begin{tabular}{lcc}
\hline & $\begin{array}{c}\text { Frequency } \\
(\%)\end{array}$ & $\begin{array}{c}\text { Percentages } \\
(\%)\end{array}$ \\
\hline Protective measures & & \\
Facemask & 144 & $47.8 \%$ \\
Face-shield & 19 & $6.3 \%$ \\
Eye-protective glasses & 23 & $7.6 \%$ \\
Personal glasses & 15 & $5 \%$ \\
None of the above & 10 & $3.3 \%$ \\
Mask, shield, eye- & 14 & $4.7 \%$ \\
$\quad$ protective glasses & & \\
Mask, eye- & 55 & $18.3 \%$ \\
$\quad$ protective glasses & & \\
Mask, personal glasses & 20 & $6.6 \%$ \\
Preference of wearing & & \\
eye protection & & $48.5 \%$ \\
Always & 146 & $32.6 \%$ \\
Sometimes & 98 & $8.6 \%$ \\
Occasionally & 26 & $2.3 \%$ \\
Rarely & 24 & \\
Never & 7 & \\
\hline
\end{tabular}

dental management. Secondly, indirect contact with contaminated surfaces and instruments. Thirdly, by inhaling a suspended air borne virus. These aerosols and splashes are generated due to the usage of a highspeed hand piece during tooth preparation, scaling, when combined with body fluids (blood and saliva bio aerosols) [27]. Literature has demonstrated the fact that exposed mucous membranes and unprotected eyes aggravate the chances of 2019-nCoV transmission [28]. In the present study, the hypothesis that there was a significant association between ocular events and associated factors was accepted.

One of the important findings of the present study were that ocular encounter was most frequent in females [204 (67.8\%)]. This outcome can be explained by the fact that study sample comprised more of female participants compared to males. Another major finding was, that final year students exhibited greatest frequency of ocular events [123 (40.9\%)] followed by house officers [97 (32.2\%)]. A probable explanation to this finding is, that fourth year students and house officers have limited clinical expertise, limited patient management skills and are less trained which maximizes their risk of any ocular event. Similarly, in government sector more ocular events were reported which are attributed to the fact that government setups might not be sufficiently equipped with protective resources like PPE, therefore showing increased ocular incidence. Number of patients treated each day also had a significant role in ocular events. Conclusion of the present study revealed that those who treated $\leq 4$ patients per day reported the highest work-related ocular events because most of them were final year students or newly graduates. It is speculated less experience in handling patients and incorrect dental posture may have contributed to this outcome.

PPE refers to combined use of protective clothing, gloves, goggles, face shields, face masks or any other form of equipment designed to protect wearer from injury or spread of illness [29]. In present study 
[144 (47.8\%)] participants stated the use of protective measure like facemask alone in routine. Whereas only $14(4.7 \%)$ reported the combined use of complete PPE which includes face mask, face shield, goggles, gloves and protective clothing. These findings are in agreement with literature which described non-compliance in dental professionals against use of proper PPE as per guidelines of Centers of Disease control and prevention [29, 30]. Barriers reported by dentists towards use of protective measures include unavailability of PPE, high PPE cost and use of PPE as effort taking.

Another major finding in the present study was that, the prevalence of ocular encounter was high $(47.8 \%)$, these findings are in agreement with the study by Azodo et al. which was reported at $72.3 \%$ [31]. On the contrary, a very low prevalence of $13.3 \%$ was reported by Pavicin et al. [4]. Nearly $2 \%$ of participants never wore any protection as reported in this study which is less in contrast to a Saudi study in which $15 \%$ of respondents reported that they never used any kind of protective equipment, these findings were due to lack of education among dentists in this regard [2]. Most of the common risk factors were reported as incorrect posture which has been confirmed by many studies previously [32, 33]. A recent study among oral and maxillofacial surgeons reported the prevalence of ocular exposure at $32.2 \%$, which is lower compared to present study findings. A possible explanation for these findings may be derived from the fact that, in the present study, dentists from all specialties were included who perform a variety of aerosol-generating procedures [34].

The findings of the current study suggest that the most common foreign body encountered by dentists was saliva splash (26.2\%), whereas in the study among dental surgeons by Azodoet and Ezeja et al. a high percentage was obtained for foreign body encounter (37.8\%), followed by foreign body and splash (22.3\%) and least for splash only (12.2\%) [10]. In the present study, scaling procedure showed $34.2 \%$ ocular events, compared to $72.0 \%$. As shown among dental surgeons in Southern Nigeria. Most of the dentists reported that the predominant action taken after the encounter of a foreign body was to rinse the eye with water [239 (79.4\%)] which may have helped to give relief to the affected individual. In our study those who were not using eye protection reported greater prevalence of ocular events, these findings are in agreement with the study by Adil et al. [34]. A German study reported that ocular encounters are twice more likely in dental students than practicing dentists which indicates that an increase in work experience increases the expertise of handling instruments and patient management [35].

CDC has suggested numerous preventive measures to avoid spread of 2019-nCoV, which can provide good vision while maintaining integrity of ocular health. This includes use of eye protection like goggles and eyeglasses in addition to facemasks to ensure that the eyes, nose and mouth are protected against exposure to respiratory secretions and oral splashes. Use of eye protection like protective glasses lessens risk of eye damage and cross-infection while performing dental procedures $[30,36]$.

It is essential to educate dentists, which will enable them and increase preparedness towards these types of incidences so that a decline in the prevalence of ocular events can be observed. The need for eye protection is emphasized in the ongoing pandemic of 2019-nCoV. Therefore, the use of safety glasses and goggles and over specs are recommended while performing the dental treatment. Protection against aerosols is through the usage of full-face or halfmask respirators, powered respirators, disposable masks [25]. Protection of the eye must be prioritized during dental treatments to avoid splashes which include aerosols generating procedures or activities with prolonging face-to-face contact or close contact with the potentially infected individual. There are powered air-purifying respirators or full-face respirators containing eye protection that should be incorporated in the dental settings. Proper use of PPE alone is not sufficient to reduce such incidence. Use of appropriate cleaning techniques and disinfection strategies must be employed. Pre-procedural mouthwash using chlorhexidine and use of rubber dam are recommended to decrease viral load in oral fluids. High-volume evacuation must also be incorporated in order to reduce aerosol [37]. E-mail: The possible limitations are subjective findings, based on opinions of an individual. Moreover, respondents in such type of study design do not feel comfortable in being honest and are less confident for any given answer because of lack of memory. Furthermore, these survey types have a low validity rate. However, the conclusions of the present study may lead to further probing and investigation on the matter of ocular injury with COVID-19 transmission with a better and comprehensive study design.

Due to characteristics of dental settings the chances of cross-infection are much greater between patients and dentists, therefore prompt infection control protocols must be taken in urgent consideration. The 
comprehensiveness of the ocular events concerning the foreign body and splash in this study will be an obvious justification for further studies.

\section{Conclusion}

In the present study, occurrence of ocular injuries was high compared to facial injuries and these outcomes were directly related to dental expertise and experiences. Appropriate measures should be adopted to minimize the risk of disease transmission and COVID-19 through the eye among practicing dentists

\section{Acknowledgment}

The authors extend their appreciation to the Deanship of Scientific Research at King Saud University for funding this work through research group no. RGP-1438-024.

\section{Author contributions}

WA and QA: Conceptualization, Methodology, Supervision, Investigation, Software, Data curation, Writing - original draft.

$\mathrm{AA}, \mathrm{OM}$ and $\mathrm{FV}$ : Investigation, Questionnaire design and distribution, Data curation, Writing and revision of manuscript.

$\mathrm{AB}$ and QA: Data collection, Data analyses, Data inference.

FV, OM and AB: Questionnaire design, Questionnaire validity, Data curation, Manuscript writing.

\section{Conflict of interest}

The authors declare no conflict of interest

\section{References}

[1] Ahn JY, Ryoo HW, Park JB, Moon S, Cho JW, Park DH, et al. Epidemiologic characteristics of work-related eye injuries and risk factors associated with severe eye injuries: a registry-based multicentre study. 2020;27(2):105-14.

[2] Alsabaani NA, Awadalla NJ, Abu Saq IH, Abualiat ZM, Alshahrani MA, Alqahtani AM, et al. Occupational ocular incidents in dentists: a multicentre study in southwestern Saudi Arabia. 2017;67(6):371-7.

[3] Kalenderian E, Obadan-Udoh E, Maramaldi P, Etolue J, Yansane A, Stewart D, et al. Classifying adverse events in the dental office. 2017.
[4] Pavičin IS, Lovrić Ž, Çeshko AZ, Vodanović MJASC. Occupational Injuries among Dentists in Croatia. 2020; 54(1):51.

[5] Ekmekcioglu H, Unur MJJoIUFoD. Eye-related trauma and infection in dentistry. 2017;51(3):55.

[6] Siva Naga Yasaswi C, Prasanthi N, Simha BV, Dabburi T, Harish Chowdary K, Chand YSJJJoMR. Occupational hazards in dentistry and preventing them. 2018;5(2):60-7.

[7] Hammouri I, Mustafa T, Al-Weshah M, Al Adwan L, Abo Rabbaie HJJotRMS. Eye Care Guidelines for Dentists. 2017;102(5512):1-8.

[8] Dagher J, Sfeir C, Abdallah A, Majzoub ZJIjod. Infection control measures in private dental clinics in Lebanon. 2017;2017.

[9] Basnet A, Basnet PS, Lamichhane RS, Shrestha PJJoCMC. Awareness, Knowledge and Willingness Regarding Eye Donation among Dental Students of Kist Medical College and Teaching Hospital, Lalitpur, Nepal. 2019;9(2):47-53.

[10] Azodo CC, Ezeja EBJAoO, Medicine E. Work-related ocular events among Nigerian dental surgeons. 2015;27(1).

[11] ALBDOUR MQ, Othman EFJPO, Journal D. EYE SAFETY IN DENTISTRY-A STUDY. 2010;30(1).

[12] Nejatidanesh F, Khosravi Z, Goroohi H, Badrian H, Savabi OJIjopm. Risk of contamination of different areas of dentist's face during dental practices. 2013;4(5):611.

[13] Izzetti R, Nisi M, Gabriele M, Graziani FJJoDR. COVID-19 transmission in dental practice: brief review of preventive measures in Italy. 2020:0022034520920580.

[14] Ho D, Low R, Tong L, Gupta V, Veeraraghavan A, Agrawal RJOi, et al. COVID-19 and the ocular surface: A review of transmission and manifestations. 2020;28(5):726-34.

[15] Checchi V, Bellini P, Bencivenni D, Consolo UJIdj. COVID19 dentistry-related aspects: a literature overview. 2020.

[16] Veena H, Mahantesha S, Joseph PA, Patil SR, Patil SHJJoi, health p. Dissemination of aerosol and splatter during ultrasonic scaling: a pilot study. 2015;8(3):260-5.

[17] Meng L, Hua F, Bian ZJJoDR. Coronavirus disease 2019 (COVID-19): emerging and future challenges for dental and oral medicine. 2020;99(5):481-7.

[18] Consolo U, Bellini P, Bencivenni D, Iani C, Checchi VJIJoER, Health P. Epidemiological Aspects and Psychological Reactions to COVID-19 of Dental Practitioners in the Northern Italy Districts of Modena and Reggio Emilia. 2020;17(10):3459.

[19] Kampf G, Todt D, Pfaender S, Steinmann EJJoHI. Persistence of coronaviruses on inanimate surfaces and their inactivation with biocidal agents. 2020;104(3):246-51.

[20] Omidi L, Moradi G, Sarkari NMJW. Risk of COVID-19 infection in workplace settings and the use of personal protective equipment. 2020;66(2):377-8.

[21] Verbeek JH, Rajamaki B, Ijaz S, Sauni R, Toomey E, Blackwood B, et al. Personal protective equipment for preventing highly infectious diseases due to exposure to contaminated body fluids in healthcare staff. 2020(4).

[22] Xu X, Li Y, Cheng L, Zhou X, Ren BJIJOS. Transmission routes of 2019-nCoV and controls in dental practice. 2020;12(9).

[23] Raosoft I. Sample size calculator. 2004. 2016.

[24] Brosius F. SPSS 21: MITP-Verlags GmbH \& Co. KG; 2013.

[25] Arvind R, Roma MJJoOI, Infection. Risk of eye infections in dental personnel and the need for its prevention: a case report. 2020;10(1):1-4.

[26] Ge Z-y, Yang L-m, Xia J-j, Fu X-h, Zhang Y-zJJoZU-SB. Possible aerosol transmission of COVID-19 and special precautions in dentistry. 2020:1-8. 
[27] Epstein JB, Chow K, Mathias RJTLID. Dental procedure aerosols and COVID-19. 2020.

[28] Kaiti RJEO. Personal Protective Equipment for Occupational Safety and Ocular Health-A Review. 2020;11:61-6.

[29] Food U, respirators DAJN, masks sf. Personal protective equipment for infection control. 2015.

[30] Dehghani F, Omidi F, Yousefinejad S, Taheri EJW. The hierarchy of preventive measures to protect workers against the COVID-19 pandemic: A review. 2020(Preprint):1-7.

[31] Azodo CC, Ezeja EBJBOH. Ocular health practices by dental surgeons in Southern Nigeria. 2014;14(1):115.

[32] Vodanović M, Sović S, Galić IJAsC. Profesionalni zdravstveni problemi med*ju stomatolozima u Hrvatskoj. 2016;50(4):310-20.

[33] Moodley R, Naidoo S, van Wyk JJJooh. The prevalence of occupational health-related problems in dentistry: A review of the literature. 2018;60(2):111-25.
[34] Aydil BA, Benlidayi ME, Kocaelli H, Dogancali GE, Genc AJJoS, Oral, Surgery M. Ocular injuries among oral and maxillofacial surgeons: Have high risk or not? An overview of a two-centered experience. 2020.

[35] Wicker S, Rabenau HFJIaoo, health e. Occupational exposures to bloodborne viruses among German dental professionals and students in a clinical setting. 2010;83(1):77.

[36] CDC. Guidance for Dental Settings: Interim Infection Prevention and Control Guidance for Dental Settings During the Coronavirus Disease 2019 (COVID-19) Pandemic 2020 [Available from: https://www.cdc.gov/coronavirus/2019ncov/hcp/dental-settings.html.

[37] Arsenault P, Tayebi AJJotMDS. Eye Safety in Dentistry and Associated Liability. 2016;64(4):12. 